\title{
Closed-loop focal plane wavefront control with the SCExAO instrument
}

\author{
Frantz Martinache ${ }^{1}$, Nemanja Jovanovic $^{2,3}$, and Olivier Guyon ${ }^{2,4,5}$ \\ ${ }^{1}$ Laboratoire Lagrange, Université Côte d'Azur, Observatoire de la Côte d'Azur, CNRS, Parc Valrose, Bât. H. Fizeau, 06108 Nice, \\ France \\ e-mail: frantz.martinache@oca.eu \\ 2 National Astronomical Observatory of Japan, Subaru Telescope, 650 North A'Ohoku Place, Hilo, HI 96720, USA \\ 3 Department of Physics and Astronomy, Macquarie University, 2109 Sydney, Australia \\ ${ }^{4}$ Steward Observatory, University of Arizona, Tucson, AZ 85721, USA \\ 5 College of Optical Sciences, University of Arizona, Tucson, AZ 85721, USA
}

Received 11 March 2016 / Accepted 29 April 2016

\begin{abstract}
Aims. This article describes the implementation of a focal plane based wavefront control loop on the high-contrast imaging instrument SCExAO (Subaru Coronagraphic Extreme Adaptive Optics). The sensor relies on the Fourier analysis of conventional focal-plane images acquired after an asymmetric mask is introduced in the pupil of the instrument.

Methods. This absolute sensor is used here in a closed-loop to compensate for the non-common path errors that normally affects any imaging system relying on an upstream adaptive optics system. This specific implementation was used to control low-order modes corresponding to eight zernike modes (from focus to spherical).

Results. This loop was successfully run on-sky at the Subaru Telescope and is used to offset the SCExAO deformable mirror shape used as a zero-point by the high-order wavefront sensor. The paper details the range of errors this wavefront-sensing approach can operate within and explores the impact of saturation of the data and how it can be bypassed, at a cost in performance.

Conclusions. Beyond this application, because of its low hardware impact, the asymmetric pupil Fourier wavefront sensor (APFWFS) can easily be ported in a wide variety of wavefront sensing contexts, for ground- as well space-borne telescopes, and for telescope pupils that can be continuous, segmented or even sparse. The technique is powerful because it measures the wavefront where it really matters, at the level of the science detector.
\end{abstract}

Key words. instrumentation: adaptive optics - methods: data analysis - techniques: high angular resolution techniques: interferometric

\section{Introduction}

Several approaches to high contrast imaging have now clearly demonstrated the power of focal-plane based image analysis. Most prominently, non-redundant aperture masking (NRM) interferometry (Tuthill et al. 2000), relying on interferometric calibration tricks in the focal plane has led to high contrast detections (of the order of 1000:1) in a regime of angular separation (typically between 0.5 and a few $\lambda / D$ ) that is still unmatched in practice by techniques like coronagraphy (Kraus \& Ireland 2012; Sallum et al. 2015). As the generation of extreme adaptive optics (XAO) instruments is coming online, more advanced wavefront control schemes developed in the context of space-borne coronagraphy like speckle nulling (Bordé \& Traub 2006) or the general framework of electric field conjugation (Give'On 2009) are being ported on-sky (Martinache et al. 2014; Cady et al. 2013). Nevertheless, it remains remarkable that such a venerable approach (the original masking idea by Fizeau was actually first tested in the 1870s), has remained relevant for well over a century. This is really a tribute to the deep understanding that interferometry has brought to the process of image formation.
More recently, it has been shown that the same selfcalibrating tricks used in masking interferometry could in fact be applied to regular (i.e. unmasked) images, assuming AOcorrection with residual wavefront errors $\leq 1$ radian RMS. The notion of closure-phase (Jennison 1958), was indeed generalized and shown to be a special case of a wider family of self-calibrating observable quantities coined kernel-phases (Martinache 2010), since they form the basis for the null-space (or kernel) of a linear operator. This generalization also opened the way for a focal-plane-based wavefront sensing approach, relying this time on the eigen-phases of the same linear operator. While this problem is generally degenerate, one way to break this degeneracy proved to be simple, and involved masking a small but non-negligible fraction of the pupil to introduce some level of asymmetry. The principles of this asymmetric pupil Fourier wavefront sensor (APF-WFS) were described by Martinache (2013), and exploited by Pope et al. (2014) to show how it could be used, for instance, to cophase a segmented mirror. This paper further expands on the possible applications of this wavefront sensor, since it has now been implemented as part of the SCExAO instrument (Jovanovic et al. 2015b), 

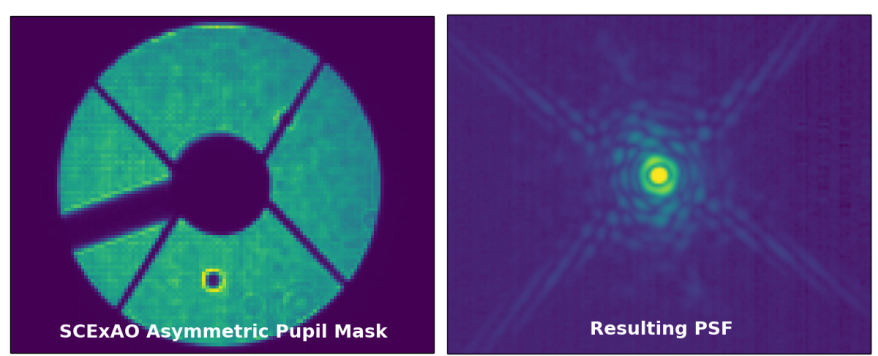

Fig. 1. Images of the pupil (left) and the focal plane (right) acquired by the SCExAO internal science camera. In addition to the four Subaru telescope spiders, the thick arm visible on the left hand side of the pupil introduces the asymmetry required for the wavefront sensing technique. The thick dot visible in the bottom pupil quadrant is induced by a dead actuator on the DM. In the focal plane, the presence of this asymmetry results in an additional set of diffraction spikes along a direction that is perpendicular to that of the arm and a lumpier first diffraction ring.

to compensate for a non-common path error unseen by its upstream pyramid wavefront sensor.

\section{Implementation of closed-loop wavefront control}

\subsection{Theoretical principle of APF-WFS}

The APF-WFS method relies on the analysis of the Fourier properties of an AO-corrected image acquired after an asymmetric hard-stop mask has been placed in the pupil. The SCExAO instrument is equipped with two of these masks with the asymmetric feature at distinct position angles, so that every part of the instrumental pupil can be accounted for. A rotation wheel, located in a plane conjugated with the pupil of the instrument (see Fig. 3 of Jovanovic et al. 2015b) makes it possible to move the masks in and out of the beam as required by the observer.

Figure 1 shows an image of one of the asymmetric masks in the pupil and the point spread function (PSF) it produces. Combined with the use of the 2000-element deformable mirror (DM), this simple alteration of the pupil is a powerful tool used to control the low-order aberrations of the instrument PSF. All images featured in this paper were acquired using an $H$-band filter, centered on wavelength $1.65 \mu \mathrm{m}$ and with an effective bandwidth of $0.3 \mu \mathrm{m}$. The pixel scale of the internal science camera is $12.1 \mathrm{mas}$ per pixel which, for this wavelength, provides a sampling better than Nyquist.

It was shown that, in the low-aberration regime, typical of what is left over after a first layer of AO correction is applied, the phase $\Phi$ measured in the Fourier transform of an image $\mathbf{I}$ and the instrumental pupil phase $\varphi$ are linearly related. On the internal calibration source, unaffected by atmospheric turbulence, the Strehl of images used in this study (such as during the calibration) is typically of the order of $80 \%$. On-sky, since the results featured here were acquired before the XAO loop is closed, the Strehl is significantly lower, of the order of $50 \%$, which is a sufficiently good starting point for approximation to be valid.

The target phase information, associated with the spatial structures of the observed object $\Phi_{0}$, is also present in the Fourier plane and simply adds to this instrumental Fourier phase. When wavefront aberrations are low (below $\sim 1$ radian), the classical image-object convolution relation

$\mathbf{I}=\mathbf{O} * \mathbf{P S F}$,
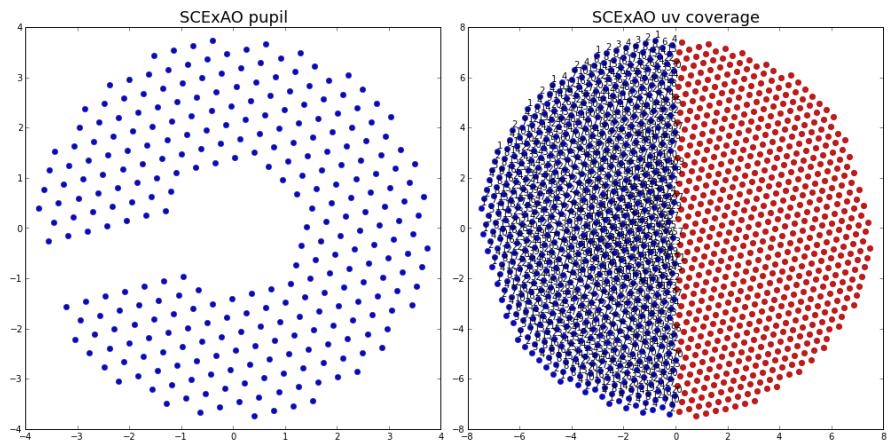

Fig. 2. Discrete model of the asymmetric pupil mask used for the calibration of the non-common path error in SCExAO. The pupil is discretized into a 292-element vector that projects onto a set of 675 equivalent interferometric baselines (or UV points) in the Fourier domain. The linear transformation that relates the wavefront to the phases measured in the Fourier transform of an image is entirely determined from this model. The presence of the asymmetry in the pupil ensures that an inverse relation for this phase-transfer matrix exists.

can therefore be reformulated, if one works with the phase part of the Fourier transform of this image as follows:

$\Phi=\Phi_{0}+\mathbf{A} \times \varphi$

where $\mathbf{A}$ is an operator that describes the way the pupil phase $\varphi$ propagates into the Fourier-plane.

When observing a point source, for which $\Phi_{0}=0$ (or if the object is known), this relation can be inverted if one introduces an asymmetry in the pupil (Martinache 2013). A direct focal plane image, with only a small amount of additional diffraction generated by the pupil asymmetry (see Fig. 1), can therefore serve as a wavefront sensor.

To determine the structure of the operator $\mathbf{A}$, a discrete representation of the instrument pupil needs to be built - including the asymmetric mask - following a regular grid with a step such that the sampling density is reasonably representative of the original pupil. Then the way this discrete model projects into equivalent interferometric baselines in the Fourier plane needs to be studied. The model currently used on SCExAO is provided in Fig. 2. It reduces the masked pupil to a 292-component vector that projects onto a 675-element vector in the Fourier domain. The phase transfer matrix $\mathbf{A}$ that establishes the mapping between the two spaces $(\Phi=\mathbf{A} \times \varphi)$ is calculated using the PYSCO software, which is used for wavefront sensing, as well as for kernel-phase data analysis of diffraction limited images.

The presence of the asymmetry in the pupil ensures that an inverse relation for this phase transfer matrix exists, and can be used to infer the pupil-phase vector $\varphi$ from the Fourier phase $\Phi$, using the relation

$\varphi=\mathbf{A}^{+} \times \Phi$,

where $\mathbf{A}^{+}$is a Moore-Penrose pseudoinverse of the phasetransfer matrix $\mathbf{A}$, computed after rejecting modes associated with low singular values. The geometry of the asymmetric feature of the mask used for this work is not the result of an optimization and simply follows the shape used in the concept paper of Martinache (2013). A smaller asymmetric feature would be expected to result in a lower sensitivy but a systematic study of the sensitivity impact of the geometry of the asymmetry has yet to be done. In the mean time, the curious reader can check the experimental work of Pope et al. (2014), which shows that, in 

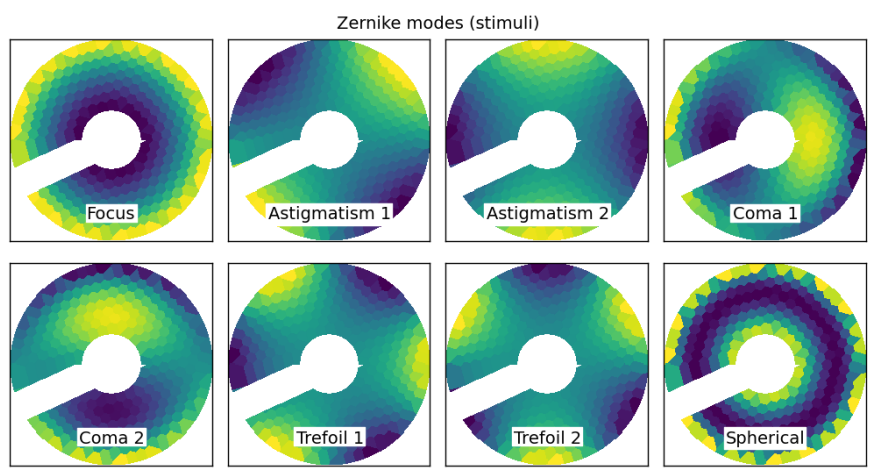

Fig. 3. The eight Zernike modes controlled by the SCExAO implementation of the APF-WFS. Sorted by a Noll index, these modes are (from left to right and top to bottom): Z4: focus, Z5: oblique astigmatism, Z6: vertical astigmatism, Z7: horizontal coma, Z8: vertical coma, Z9: vertical trefoil, Z10: oblique trefoil and Z11: primary spherical.

the case of a segmented aperture, the technique remains effective, even with a minimum of asymmetry (a single segment of the aperture) and suddenly breaks, if no asymmetry is present at all, validating the mathematical model this approach relies on.

\subsection{Integrating a real system}

The case featured in Martinache (2013) was somewhat idealized. Working on monochromatic images, and with a perfect DM, it was able to exactly generate the wavefront correction determined by the analysis. To deploy this method on an actual closed-loop system is not as direct and requires us to take into account the actual properties of the DM, such as the response curve of the actuators, their influence functions, as well as a careful mapping of these DM actuators on the instrument pupil (Blain 2013). While possible, this type of model is very prone to errors and its maintenance is demanding because of small changes in the internal instrument alignment that are induced by temperature drifts or after a telescope slew.

AO systems in operation usually choose to rely on a more pragmatic approach that encapsulates this kind of model in a transparent manner: individual DM actuators or groups of actuators (pre-defined modes) are sequentially excited and the system response is recorded and assembled in a matrix. Filtering of the noisy modes before inversion (using SVD or similar procedures) leads to the obtention of a control matrix that can be used to directly multiply an input vector made of the wavefront sensor raw input data.

This pragmatic approach is the one that was retained for this implementation of APF-WFS. The control software was designed to sense and control eight low-order Zernike modes (Zernike 1934) that correspond to classical optical aberrations: focus, two terms of coma, astigmatism and trefoil, and spherical aberration. Figure 3 shows how these modes map on the discrete pupil model used to describe the instrument.

\subsection{Properties of the phase transfer model}

To better appreciate the impact of the phase-transfer model, one can look at the effect of the projection in the Fourier plane and then back in the pupil plane using the linear relations of Eqs. (2) and (3). The original phase $\varphi$ becomes

$\varphi^{\prime}=\mathbf{A}^{+} \times \mathbf{A} \times \varphi$

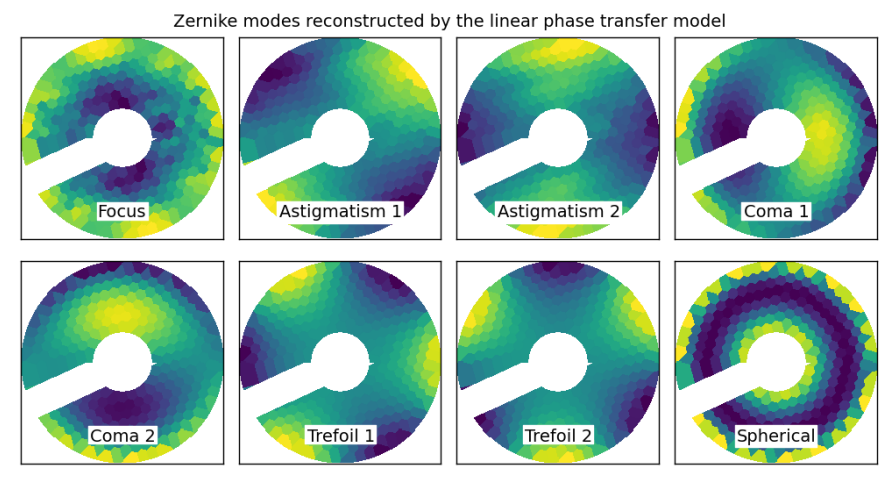

Fig. 4. The eight Zernike modes reconstructed by the linear model when 150 out of the 291 modes are kept in the computation of the pseudoinverse of the phase transfer matrix $\mathbf{A}^{+}$.

Depending on the number of modes kept in the determination of the pseudo-inverse $\mathbf{A}^{+}$of the phase-tranfer matrix, the reconstruction goes from perfect (if all modes are preserved) to very partial (if few modes are preserved). The modes discarded correspond to low singular values which, for a given level of signal-to-noise in actual data, would result in amplified noise. On SCExAO, for the control of these eight low-order modes, 150 out of the 291 available modes are maintained in the computation of the pseudo inverse. Under these conditions (see Fig. 4), the reconstruction appears satisfactory, and confirms that the technique can indeed be used to control low-order modes, assuming that the linear model holds (and that aberrations are small).

\subsection{Calibration}

The calibration procedure for this implementation of the APFWFS follows the linear control framework. After the asymmetric mask has been inserted, one image labeled as reference is acquired, followed by a sequence of images acquired after a Zernike mode of appropriate amplitude has been applied to the DM. Figure 4 features one of these calibration data-set, which was acquired with the focal camera of SCExAO on its internal calibration source (super-continuum laser) using a standard $H$-band filter for a $30 \mathrm{~nm}$ RMS deformation of the DM. We note that this displacement actually translates into a $60 \mathrm{~nm}$ wavefront amplitude modulation (the DM being a reflective system).

Each image is recentered and windowed by a super-gaussian function that filters out high-spatial frequencies, and reduces the impact of detector readout noise. It is then Fourier-transformed and the Fourier-phase is extracted according to the sampling model featured in the right hand side of Fig. 2 to populate a vector $\Psi$.

To each Zernike mode, a Fourier-phase vector $\Phi_{i}$ can be associated after subtracting the phase $\Psi_{\text {ref }}$ measured in the initial or reference state:

$\Phi_{i}=\Psi_{i}-\Psi_{\text {ref }}$

The wavefront associated with this Fourier-phase signature can be recovered using the pseudo-inverse $\mathbf{A}^{+}$previously computed and applying Eq. (3). This wavefront in radians can, in turn, be converted into a DM displacement map (in microns) after being multiplied by the proper $\lambda / 4 \pi$ scaling factor; where $\lambda$ is the wavelength expressed in microns and $4 \pi$ contains the $\times 2$ factor caused by the reflection. Figure 6 features an example of experimentally recovered modes. One will observe that the reconstruction from the Fourier analysis of actual images appears 

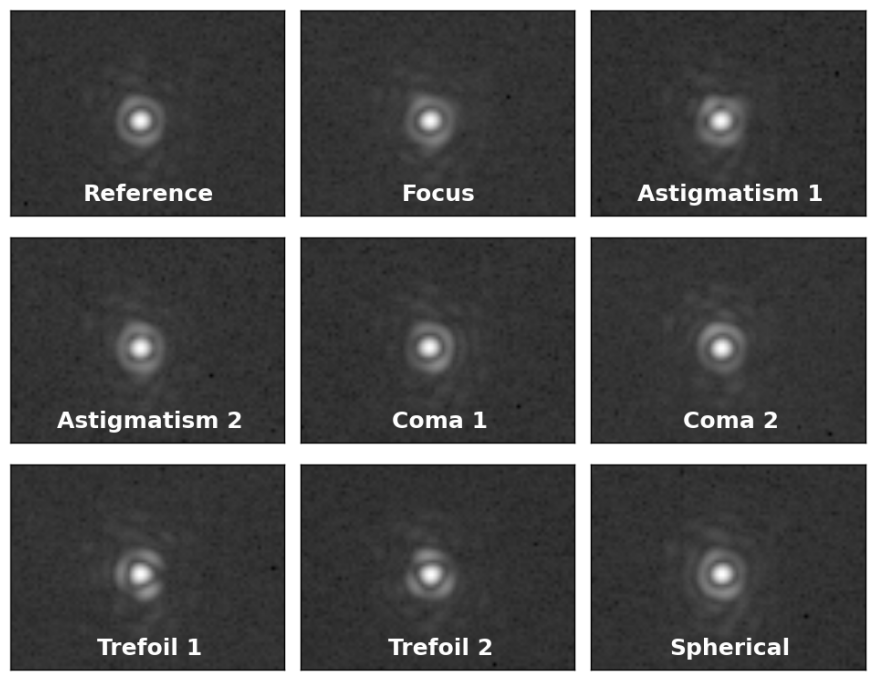

Fig. 5. Calibration data for the APF-WFS acquired by the SCExAO science camera. Top left: the reference PSF, acquired with the system in its starting state. From left to right and top to bottom: the PSF after the corresponding Zernike mode has been applied. A non-linear scale is used to better show the impact of a $30 \mathrm{~nm}$ RMS DM modulation.
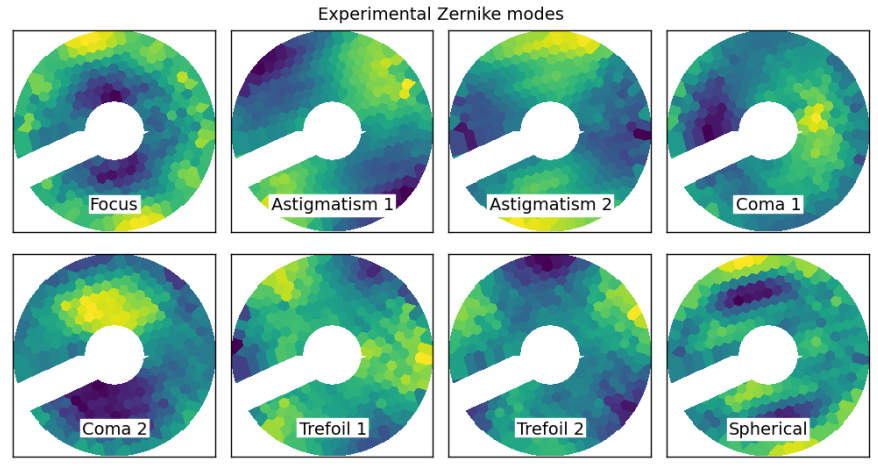

Fig. 6. Experimentally recovered Zernike modes. Save for the spherical aberration, it can be seen that the modes extracted from the analysis of the images of Fig. 5 reproduce the features expected after looking at the theoretically reconstructed modes presented in Fig. 4.

visually satisfactory. Differences in the reconstruction with the modes plotted in Fig. 4 can be attributed to imperfections of the pupil discretization model (see Fig. 4 of Martinache 2013), combined with practical subtleties like the fact that the dynamical range on the camera is limited and that classical noises, such as photon and readout, do apply.

To complete this description of the general aspect of the modes with a quantitative estimate of the quality of the reconstruction, Fig. 7 directly compares the experimental reconstruction $E$ to the theoretical Zernike modulation $T$, by plotting the local deduced DM displacement against its predicted value. One can confirm that all modes are not equally reproduced by the analysis and that, for this experimental setup, the sensor is most sensitive to astigmatism (for which the correlation coefficient is the strongest) and not particularly suited to the sensing of spherical aberration. Table 1 accompanies this figure and provides the value of the Pearson product-moment correlation coefficient for all modes:

$r=\frac{\operatorname{cov}(E, T)}{\sigma_{E} \sigma_{T}}$.
Table 1. Pearson product-moment correlation coefficient of the experimentally reconstructed mode $E$ with their theoretical counterpart $T$.

\begin{tabular}{ll}
\hline \hline Zernike mode & quality \\
\hline Z4 (focus) & 0.855 \\
Z5 (astigmatism 1) & 0.949 \\
Z6 (astigmatism 2) & 0.925 \\
Z7 (coma 1) & 0.820 \\
Z8 (coma 2) & 0.862 \\
Z9 (trefoil 1) & 0.827 \\
Z10 (trefoil 2) & 0.854 \\
Z11 (spherical) & 0.522 \\
\hline
\end{tabular}

Table 1 also shows that, with a correlation coefficient $\sim 0.5$, spherical aberration is significantly less well reconstructed than the other modes that exhibit a correlation coefficient $>0.8$. The specificity of the response to spherical aberration is, however, not an intrinsic limit of the sensing approach and can, in fact, simply be explained by the 2D geometry of this aberration and how it fits within the footprint of the Subaru Telescope and its large $(\sim 30 \%)$ central obstruction. By looking for instance at Fig. 3, we can see that the donut shape of the spherical aberration results in a pretty uniform distribution of the phase, which only varies near the inner and outer edges of the pupil. The basis of Zernike polynomials is defined for a complete circular aperture: quoted amplitudes correspond to a given wavefront RMS over the entire circular aperture. For the spherical aberration, the presence of the central obstruction naturally filters out a lot of the effect of the spherical aberration. This is further confirmed after a close examination of scatter plots of Fig. 7: whereas all Zernike stimuli (along the horizontal axis of the plots) have the same amplitude, we can see that the resulting range of local DM displacement (corresponding to the horizontal spread of the data points) is appreciably shorter for the spherical aberration than it is for the other modes.

These experimentally obtained pupil-phase modes $\varphi_{i}$ are stored in a $8 \times 291$ matrix $\mathbf{Z}$, referred to as the response matrix. In practice, unless the DM registration were to change in a dramatic manner, the calibration is quite robust: a response matrix acquired using the internal calibration source can be very well used during on-sky observations if the filter remains unchanged, and if the change of exposure time does not result in a saturated PSF core (see the discussion in Sect. 4).

On SCExAO, the acquisition of this response matrix only takes a few seconds, so it can easily be repeated if neessary after acquisition of a new target. In practice, it seems a response matrix that is acquired using the stable internal calibration source provides the best results.

\subsection{Closed-loop operation}

Just as during the calibration, focal-plane images acquired on-sky with the asymmetric mask are dark-subtracted, recentered, and windowed by a super-Gaussian function before being Fourier-transformed. After extraction of the Fourier phase, a wavefront is produced and directly projected onto the basis of modes (without subtracting the reference), to find the coefficients associated with all eight Zernike components. If the current wavefront sensor signal is $\varphi$, the instant Zernike coefficients $(\alpha)$ are the solution of $\mathbf{Z} \cdot \alpha=\varphi$. The least square solution $\left(\hat{\alpha}_{i}\right)_{i=4}^{11}$ of this system is the solution to

$\mathbf{Z}^{\mathrm{T}} \mathbf{Z} \cdot \hat{\alpha}=\mathbf{Z}^{\mathrm{T}} \varphi$ 

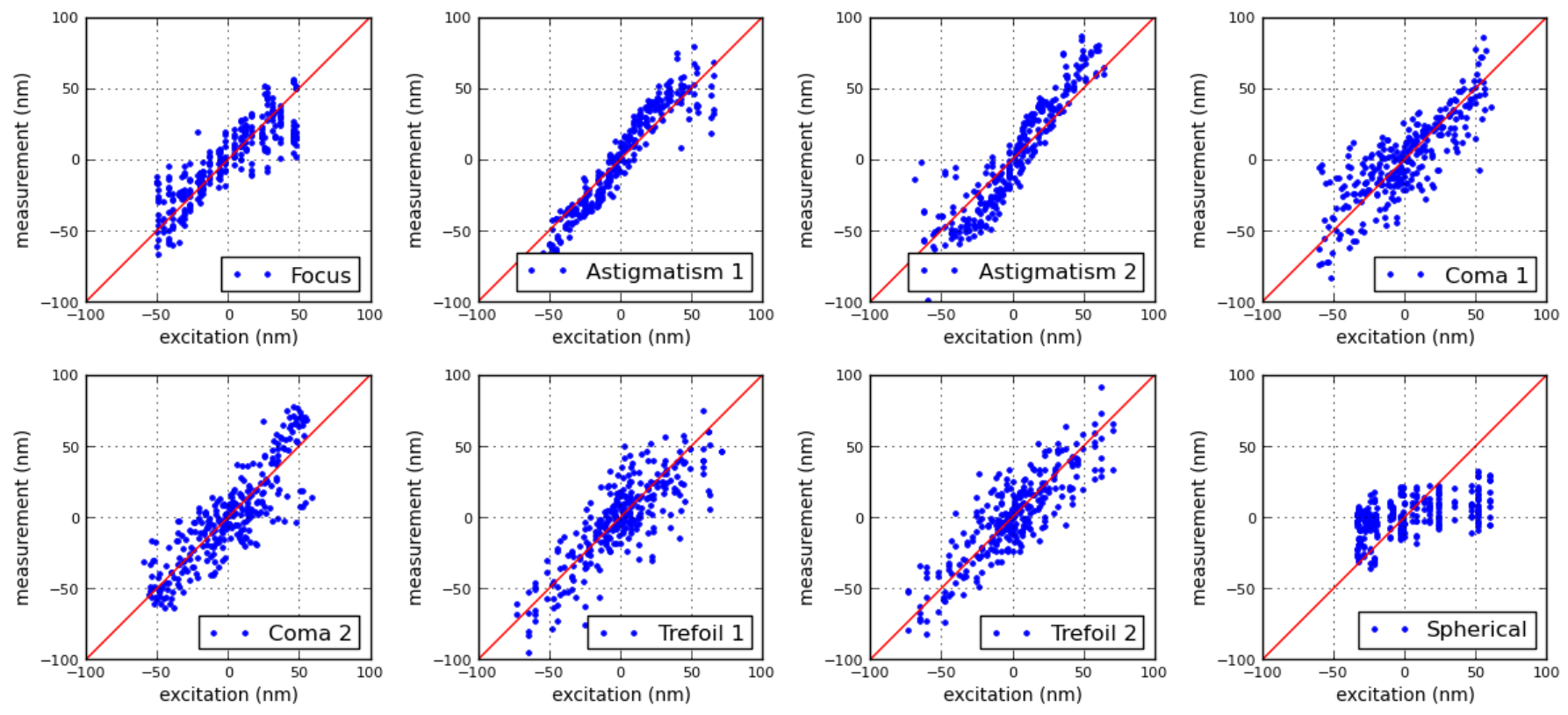

Fig. 7. Comparative quality of the modal reconstruction: for each mode, the local value of the DM displacement (in nm) for the theoretical Zernike mode of predefined amplitude (here $30 \mathrm{~nm}$, labeled as the "excitation") is plotted against its experimental measurement.
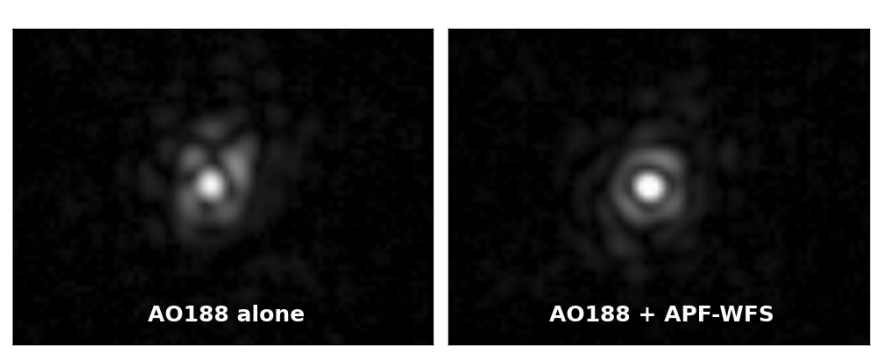

Fig. 8. Illustration of the impact of the APF-WFS. Left: $0.5 \mathrm{~ms}$ PSF acquired by SCExAO's internal science camera after the upstream AO loop has been closed. Right: identical exposure acquired $30 \mathrm{~s}$ after the APF-WFS loop has been closed. Despite residual imperfections owing to dynamic changes, the PSF quality is obviously improved.

The solution $\hat{\alpha}$ to this well-behaved system of equations is used as an input for a control loop algorithm. The loop in operation on SCEXAO implements a simple proportional controller, with a gain common to all Zernike modes with value contained between 0.05 and 0.3 , depending on the overall stability of the wavefront provided by the upstream AO. When looking at the internal calibration source, we can reliably use the highest gain. Since, for now, it is only used for a very short time (typically $\sim 15 \mathrm{~s}$ ), at the time of target acquisition to flatten the static component of the wavefront, the current implementation of the algorithm proves satisfactory. Once the non-common path error is accounted for, the asymmetric mask is taken out of the optical path and the system is ready for observation using the full pupil of the telescope.

\section{Performance}

\subsection{On-sky demonstration}

The technique was successfully deployed and proved to be effective at reducing the non-common path error during on-sky observations behind Subaru Telescope's facility AO system AO188
(Minowa et al. 2010). Figure 8 illustrates the impact of the approach, with two $500 \mu$ s exposures of the target (Altair) acquired by SCExAO's internal science camera on UT 2015-10-30.

The first image shows the PSF after the AO188 loop has been closed on the target: although it features a well-defined diffraction core, the PSF clearly exhibits some static aberrations that can be attributed to the non-common path error between AO188 and SCExAO's focal plane. The second image shows the PSF about $30 \mathrm{~s}$ after the APF-WFS loop has been closed. The gain in Strehl is low (of the order of 5\%), but the PSF is improved at where it matters most for high contrast imaging and no longer features any obvious low-order aberration signature. Residual inhomogeneity of the first diffraction ring can be attributed to a combination of instantaneous $\mathrm{AO}$ residuals combined with the effect of the asymmetric arm.

SCExAO's internal science detector is a fast but lowsensitivity detector that can acquire images at up to $170 \mathrm{~Hz}$ full-frame rate whose specifications are given by Jovanovic et al. (2015b). APF-WFS seems to exhibit sufficient sensitivity to be used in a fast closed-loop that could very well track low-order aberrations with frequencies up to a tenth of the camera frame rate.

At the moment, the goal of the loop is to calibrate the quasistatic non-common path error. The control software keeps a rolling average of the 20 last wavefront estimations, and corrects for the average of these estimations at each iteration, thus filtering vibration-induced fast varying component. Combined with the acquisition, the (non-optimized) computation of the wavefront makes the loop run at a frequency of $\sim 8 \mathrm{~Hz}$.

\subsection{Cross-talk}

Zernike polynomials (Zernike 1934) form a convenient basis to describe a wavefront within a circular aperture: designed to form an orthonormal basis, the first terms of the series happen to correspond to classical monochromatic optical aberrations like 

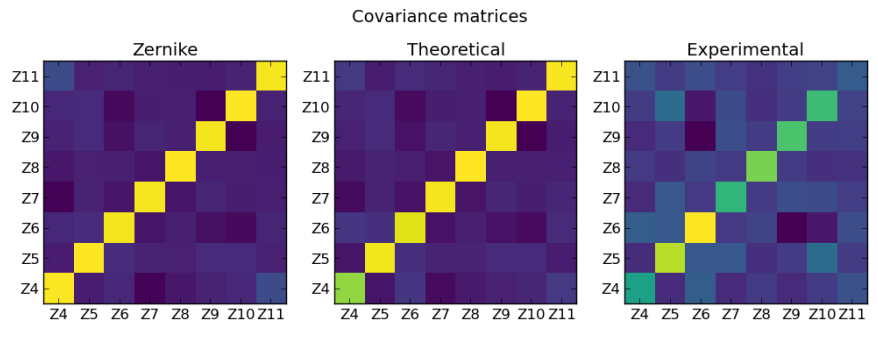

Fig. 9. Control modes inner product matrices characterizing the orthogonality properties of the implementation of the APF-WFS described in this paper on SCExAO. From left to right: 1. the mostly perfectly diagonal case of the Zernike polynomials basis; 2 . the seemingly identical inner product matrix for the theoretical modes after reconstrucion by the linear model and filtering by the SVD; and 3. the inner product matrix for the experimentally reconstructed modes. The overall diagonal allure of the latter characterizes the sensor as suited to the control of the low-order Zernike modes. Numerical values for the experimental inner products are provided in Table 2.

focus, astigmatism or coma. As previously seen, the presence of a central obstruction in the pupil makes this basis no longer perferctly orthogonal. Substitutes have been proposed (Mahajan 1981) to accomodate for the presence of this rather common feature of telescopes, but the asymmetric arm required for the wavefront sensing (see Sect. 1) would also require an adaptation.

Instead of trying to specify a new orthogonal basis perfectly adapted to our case, we have judged it more appropriate to stick to the conventional Zernike basis and verify a posteriori how orthogonal the different modes actually are. Figure 9 does this by plotting the $8 \times 8$ matrix of inner products between the eight control modes $\left(\mathbf{Z}^{\mathrm{T}} \mathbf{Z}\right)$ for three cases: the input Zernike polynomials (given in Fig. 3), the theoretical reconstruction of the linear model with 150 out of the 291 eigen modes kept in the phase transfer matrix inversion (given in Fig. 4), and the experimentally acquired modes (given in Fig. 6). An orthogonal basis will result in a perfectly diagonal inner-product matrix, whereas nonorthogonality would become manifest with strong non-diagonal components.

One can therefore observe, looking at the left hand side panel of Fig. 9, that the Zernike modes form a satisfactory, nearly orthonormal basis, with a mostly uniform diagonal and with a limited amount of cross terms standing out (except in the case of the $16 \%$ cross-correlation between focus (Z4) and spherical (Z11). For the sake of consistency with the rest of the data presented in the paper, Fig. 9 also shows in its central panel, that the modes reconstructed by the linear model reproduce most of these features, although one can observe $\sim 20 \%$ degradation of the relative strength of the focus signal. What we observe here is the effect of the filtering of low singular values in the construction of the pseudo-inverse $\mathbf{A}^{+}$as used in Eq. (4). With 150 out of the possible 291 modes kept in the construction of the pseudo-inverse, the inner product matrix for the experimentally recovered modes is also mostly diagonal.

Table 2 provides the numerical values for the experimental inner products, also graphically represented in the right hand side panel of Fig. 9. Although some of the cross terms are nonneglibible, the terms along the diagonal still dominate, indicating that a control loop that relies on this calibration dataset will reliably converge toward a state that will cancel the non-common path aberration.
Table 2. Numerical values for the experimental modes' inner-product.

\begin{tabular}{l|rrrrrrrr}
\hline \hline & Z4 & Z5 & Z6 & Z7 & Z8 & Z9 & Z10 & Z11 \\
\hline Z4 & 0.46 & -0.09 & 0.12 & -0.10 & -0.04 & -0.09 & -0.03 & 0.06 \\
Z5 & -0.09 & 0.87 & 0.09 & 0.09 & -0.08 & -0.03 & 0.19 & -0.03 \\
Z6 & 0.12 & 0.09 & 1.00 & -0.04 & 0.01 & -0.25 & -0.16 & 0.05 \\
Z7 & -0.10 & 0.09 & -0.04 & 0.57 & -0.04 & 0.05 & 0.03 & -0.02 \\
Z8 & -0.04 & -0.08 & 0.01 & -0.04 & 0.75 & -0.03 & -0.07 & -0.07 \\
Z9 & -0.09 & -0.03 & -0.25 & 0.05 & -0.03 & 0.65 & -0.03 & -0.02 \\
Z10 & -0.03 & 0.19 & -0.16 & 0.03 & -0.07 & -0.03 & 0.61 & 0.00 \\
Z11 & 0.06 & -0.03 & 0.05 & -0.02 & -0.07 & -0.02 & 0.00 & 0.11 \\
\hline
\end{tabular}
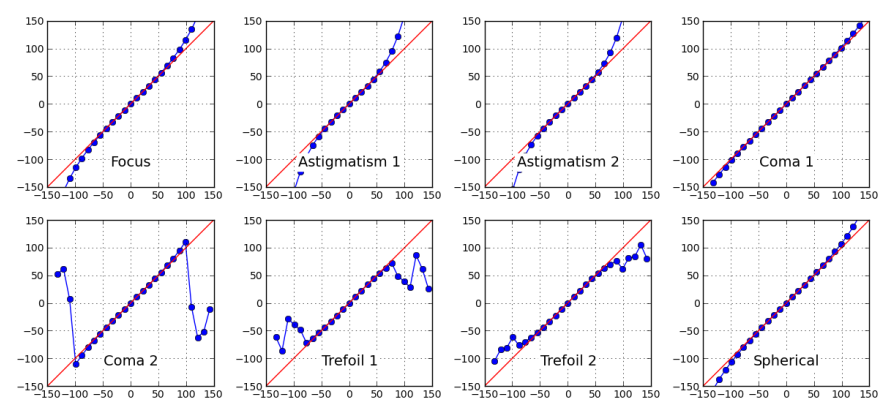

Fig. 10. Experimental response of the APF-WFS obtained on the SCExAO internal (super-continuum) source in the $H$-band. Each plot features (on the vertical axis) the reponse of the sensor to a Zernike mode of RMS amplitude that varies over a $\pm 150 \mathrm{~nm}$ range (units for both axis are in $\mathrm{nm}$ ). While nearly linear over the entire range for most modes, the sensor only exhibits a significant non-linear behavior for the coma 2, and the two trefoil modes when the DM Zernike amplitude is larger than $80-100 \mathrm{~nm}$. We note that this limit is on the DM surface, which must be doubled if refering to aberrations on the wavefront.

\subsection{Range of linear response}

As mentioned in Sect. 2.1, the APF-WFS relies on the assumption that an upstream AO correction is provided. The system is expected to deal with with small residual wavefront errors, and the calibration procedure described above, typically employs DM modulation amplitudes of $\sim 30-50 \mathrm{~nm}$. To determine the amount of aberration the technique is able to deal with, we performed a systematic exploration of the response of the sensor to stimuli of variable amplitude. The instantaneous response of the sensor is projected onto the basis of modes following the procedure outlined in Sect. 2.5. Figure 10 summarizes the results of this systematic exploration of the response of the sensor, over a $\pm 150 \mathrm{~nm}$ range of DM modulation amplitude.

Although not perfectly linear, for Z4 (focus), Z5, Z6 (astigmatism), Z7 (coma 1), and Z11 (spherical), the response remains monotonic over the entire $\pm 150 \mathrm{~nm}$ range. For Z8 (coma 2), Z9 and Z10 (trefoil 1 and 2), the response is only monotonic over the $\pm 80 \mathrm{~nm}$ modulation range beyond which the sensor cannot be used reliably. The drastic difference of response between $\mathrm{Z7}$ and Z8, which corresponds with the same type of aberration (i.e. coma), can be explained by the azimuth of the asymmetric arm in the pupil stop, oriented such that it almost entirely masks out one of the two antisymmetric bumps that are characteristic of this aberration (see for instance the top left panel of Fig. 3).

A strong non-linearity of the response is experienced when the pupil-phase peak-to-valley (P2V) wavefront becomes larger than $2 \pi$ (which results in a phase wrap). The presence of the asymmetric stop at its current azimuth essentially divides the $\mathrm{P} 2 \mathrm{~V}$ by a factor of two in the case of $\mathrm{Z7}$ (i.e. coma 1), hence 

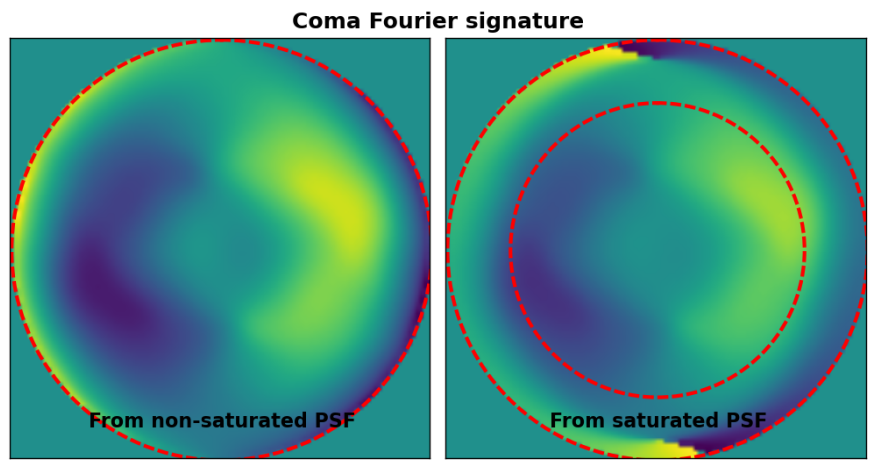

Fig. 11. Comparison of the UV-phase signature of the same amount of aberration (here coma) for a non-saturated PSF (on the left) and a saturated one (on the right). The saturation primarily affects the higher spatial frequencies of the image, corresponding to the outermost parts of the Fourier plane. In the inner region of the saturated case (within the highlighted smaller circle), the Fourier-phase signature of the aberration is similar to its non-saturated counterpart.

making the sensor able to handle twice as much coma along the horizontal axis than along the vertical axis. We note that the same effect (to a lesser extent) can also be observed when comparing $\mathrm{Z} 9$ and Z10. We can nevertheless conclude that, in the $H$-band under normal operating conditions, the sensor is able to operate linearly as long as the RMS error on either mode is less than $200 \mathrm{~nm}$ on the wavefront.

\section{Wavefront sensing from a saturated PSF}

A well AO-corrected PSF is a highly contrasted object. The proper simultaneous sampling of the core of the PSF and its diffraction rings therefore requires a detector with a large dynamical range, which is rarely compatible with a fast readout. The SCExAO internal science camera has an effective dynamical range of $\sim 10000$ counts so that, in practice, an exposure time has to be chosen that either gives access to a non-saturated PSF core (the normal operating mode of the wavefront sensing approach described thus far) or over-expose the PSF core to better see the fainter diffraction structures that surround it.

In its general form, the linear model of Eq. (2) only holds when working on non-saturated images that otherwise result in a non-translation invariant PSF. Pixels that are saturated by the bright core of the PSF can be treated as zeros, so that the effect of saturation can be modeled as a multiplication by a top-hat function that cuts off anything higher than a level imposed by the characteristics of the detector. This multiplication in the image space results in a convolution by an Airy-like function whose characteristic size is inversely proportional to the size of the saturated part of the PSF.

The effect of this convolution is expected to be most prominent in the outermost region of the Fourier plane where the phase will experience a change of sign. Figure 11 illustrates this effect by comparing the Fourier-phase signature of a specific aberration for a non-saturated PSF to that of a mildly saturated one. We can see that, while the outermost part of the Fourier-phase is obviously affected by the saturation, the innermost part of the PSF (highlighted by the smaller dashed circle) does resemble the original non-saturated case.

Figure 12 further makes this obvious by representing in a 1D plot the values of the Fourier-phase of the saturated image against the Fourier-phase of the non-saturated image. Whereas

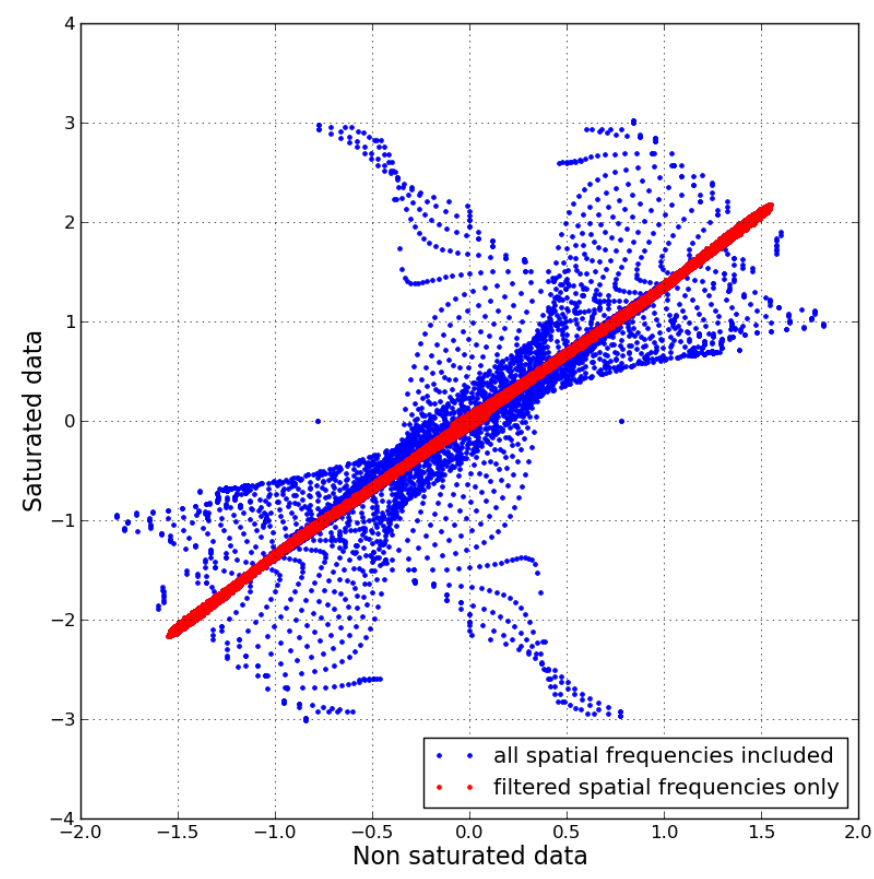

Fig. 12. 1D comparison of the phase (in radians) extracted from the Fourier plane, featured in Fig. 11, in the non-saturated case (along the horizontal axis) and in the saturated case (along the vertical axis). The blue points include the data at all spatial frequencies while the red ones correspond to the inner part of the Fourier plane only. The strong correlation observed in the latter case suggests that some of the wavefront information can be recovered from the analysis of mildly saturated data.

considered as a whole, the Fourier-phase of the saturated data appears as non-usable (the blue points are widely scattered), the inner part of this same saturated data set is strongly correlated (the red points) with the non-saturated data, suggesting that some of the wavefront information can be recovered in the saturated case, assuming that we can filter out the information coming from the largest baselines.

To account for this filtering, the Fourier-phase model can be modified, and the parts of the phase transfer matrix $\mathbf{A}$ can be discarded along with the parts of the Fourier-phase vector $\Phi$ that are filtered out. The model we tested only preserves baselines that are $70 \%$ or less than the longest baseline in the model, which corresponds to the area inscribed within the inner circle plotted in the right panel of Fig. 11. Out of the 675 original distinct UVphase samples, 330 remain with this configuration, which is still of the order of the number of modes necessary to recover the full theoretical pupil phase information (291 modes).

For the computation of the pseudo-inverse $\mathbf{A}^{+}$of this new system, 50 modes are kept. With less constraints from the UVplane, the Zernike modes are less well reconstructed, but are nevertheless recognizable, as shown in Fig. 13.

The calibration procedure introduced in Sect. 2.4 can be repeated with this new model, after which the APF-WFS is effectively able to operate in closed-loop from the analysis of saturated data, albeit with lower performance. To see how this saturation affects the sensor, the study presented in Sect. 3.3 was repeated in this new operating mode. The outcome of this study is presented in Fig. 14.

In this peculiar saturated mode, the sensor is able to operate linearly over a limited range of aberrations. As previously observed (see Sect. 3.3), it is the modes whose geometry features 

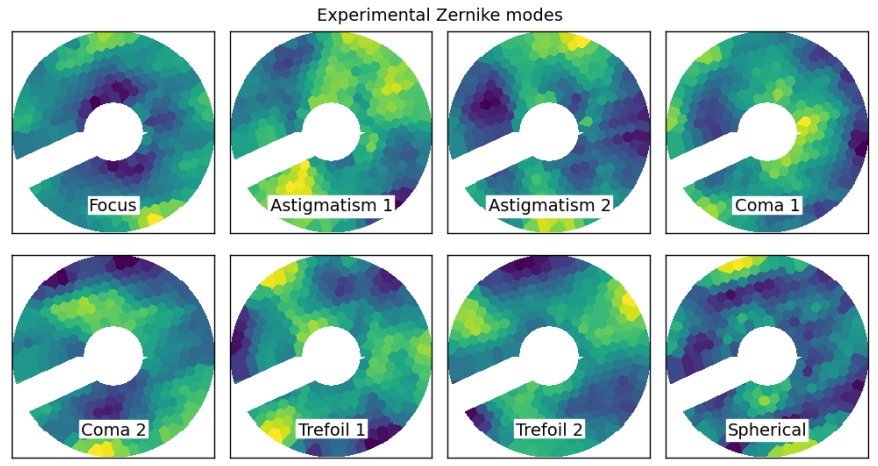

Fig. 13. Experimentally recovered Zernike modes after discarding the phase associated with the longest baselines, which are affected by saturation effects. This new series of experimental modes should be compared to the non-saturated case presented in Fig. 6.
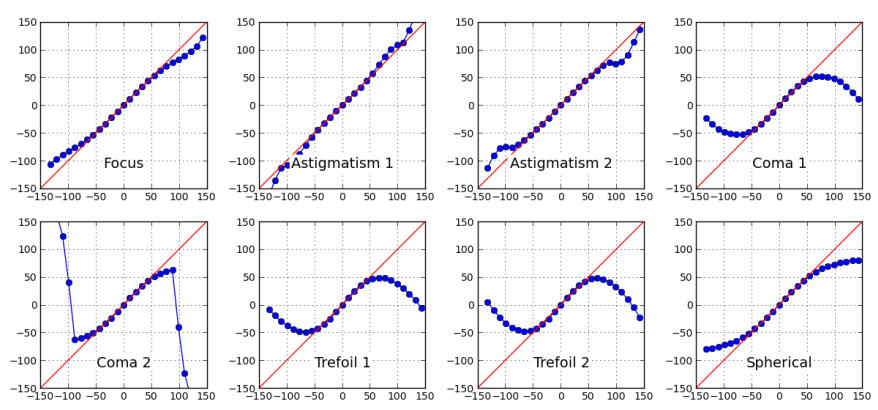

Fig. 14. Study of the response of the sensor in the saturated case to Zernike modes of varying amplitude. This series is to be compared to the case presented in Fig. 10 in the non-saturated case.

localized bumps, such as coma of trefoil, that first limit the range of aberration that the APF-WFS can account for. The APF-WFS can nevertheless operate on images whose core is saturated, over a $100 \mathrm{~nm}$ wavefront RMS range that is roughly one half of what it can achieve in the non-saturated operating regime: the general APF-WFS approach is more robust than at first expected and can be used despite the less than ideal conditions.

\section{Conclusion}

Following on from the conceptual study proposed by Martinache (2013), this paper described the implementation of the asymmetric pupil Fourier wavefront sensor as one of the wavefront control loops of the SCExAO instrument. This approach has proven capable of repeatedly accounting for the non-common path error that affects the instrument after a new telescope pointing and provides an updated zero-point for the upstream pyramid wavefront sensor currently implemented inside SCExAO.

A surprisingly simple asymmetric hard stop mask introduced in the pupil of a diffraction-limited imaging instrument is thus proving to be a powerful diagnostic tool for the control of the non-common path aberrations. The reported capture range of the technique is currently limited to a fraction of a wave (RMS $\sim \lambda / 8)$. A combination of filters of decreasing wavelengths would provide a direct way to tolerate a cruder starting point. We are currently exploring the potential of an updated algorithm that simultaneously exploits the information sampled at multiple wavelengths to extend the capture range even more, this time within the coherence length. We note that other approaches using combinations of non-redundant aperture masks (Cheetham et al. 2012, 2014) also rely on this idea to extend their capture range.

The asymmetry results in slight cosmetic degradations of the PSF. While this does impact a coronagraphic instrument, it can be tolerated in a general purpose AO-corrected imaging instrument. A very interesting feature of this image-based wavefront sensing approach is that, if multiple sources are available in a given field, the APF-WFS algorithm can be used on all sources simultaneously. Depending on the complexity of the field, the same asymmetric mask, combined with the analysis of multiple sources in one image, can be used for multi-reference wavefront sensing, opening the way to a full 3D reconstruction of the wavefront from the analysis of a single focal plane image. This very property can also be put to use on artificially introduced incoherent replicas of an on-axis PSF of tunable intensity, as described by Jovanovic et al. (2015a), thus making the use of the technique compatible with that of a coronograph that otherwise destroys the interferometric reference required for a sensible Fourier-analysis of the image, as described in this work.

The use of this wavefront control technique extends well beyond the control of low-order modes on SCExAO: this paper provides experimental evidence that the technique is actually effective where the theory predicts it should be. In an exposure that simultaneously features an unsaturated PSF core and the diffraction features at large separation with sufficient SNR, APF-WFS can be used to control an arbitrary number of modes, as was shown in the concept paper. The APF-WFS can, in fact, be easily applied in a wide variety of wavefront sensing contexts, for ground- as well space-borne telescopes, and with a pupil that can be continuous, segmented, or even sparse. APF-WFS is powerful because it measures the wavefront where it really matters, at the level of the science detector. Given its low impact on the instrument hardware, it is an option that should be given some consideration as part of any high contrast imaging instrument with wavefront control capability.

\section{References}

Blain, C. 2013, Ph.D. Thesis, University of Victoria (Canada)

Bordé, P. J., \& Traub, W. A. 2006, ApJ, 638, 488

Cady, E., Baranec, C., Beichman, C., et al. 2013, in Techniques and Instrumentation for Detection of Exoplanets VI, Proc. SPIE, 8864, 88640

Cheetham, A., Cvetojevic, N., Norris, B., Sivaramakrishnan, A., \& Tuthill, P. 2014, Optics Express, 22, 12924

Cheetham, A. C., Tuthill, P. G., Sivaramakrishnan, A., \& Lloyd, J. P. 2012, Optics Express, 20, 29457

Give'On, A. 2009, in Techniques and Instrumentation for Detection of Exoplanets IV, Proc. SPIE, 7440, 74400

Jennison, R. C. 1958, MNRAS, 118, 276

Jovanovic, N., Guyon, O., Martinache, F., et al. 2015a, ApJ, 813, L24

Jovanovic, N., Martinache, F., Guyon, O., et al. 2015b, PASP, 127, 890

Kraus, A. L., \& Ireland, M. J. 2012, ApJ, 745, 5

Mahajan, V. N. 1981, J. Opt. Soc. Am. (1917-1983), 71, 75

Martinache, F. 2010, ApJ, 724, 464

Martinache, F. 2013, PASP, 125, 422

Martinache, F., Guyon, O., Jovanovic, N., et al. 2014, PASP, 126, 565

Minowa, Y., Hayano, Y., Oya, S., et al. 2010, in SPIE Conf. Ser., 7736

Pope, B., Cvetojevic, N., Cheetham, A., et al. 2014, MNRAS, 440, 125

Sallum, S., Follette, K. B., Eisner, J. A., et al. 2015, Nature, 527, 342

Tuthill, P. G., Monnier, J. D., Danchi, W. C., Wishnow, E. H., \& Haniff, C. A. 2000, PASP, 112, 555

Zernike, v. F. 1934, Physica, 1, 689 